
\title{
R Research Soure \\ Renocardiac Syndrome Induced by Renal Ischemia and Reperfusion: Vibrational Markers
}

\author{
Gabrielle Nepomuceno \\ Universidade Federal do ABC \\ Carolina Junho \\ Universidade Federal do ABC \\ Marcela Carneiro-Ramos \\ Universidade Federal do ABC \\ Herculano Martinho ( $\nabla$ herculano.martinho@ufabc.edu.br) \\ Universidade Federal do $A B C$
}

\section{Research Article}

Keywords: Renal injury, heart morphology, electrophysiology, renocardiac syndrome

Posted Date: December 30th, 2020

DOl: https://doi.org/10.21203/rs.3.rs-134409/v1

License: (c) (i) This work is licensed under a Creative Commons Attribution 4.0 International License.

Read Full License

Version of Record: A version of this preprint was published at Scientific Reports on July 22nd, 2021. See the published version at https://doi.org/10.1038/s41598-021-93762-z. 
Renocardiac syndrome induced by renal ischemia and reperfusion: vibrational markers

Gabrielle Nepomuceno, Carolina Victoria Cruz Junho, Marcela Sorelli Carneiro-Ramos, Herculano da Silva Martinho*

Universidade Federal do ABC, Centro de Ciências Naturais e Humanas, Av. dos Estados, 5001, 09210-580, Santo André, SP, Brazil

*Corresponding Author: herculano.martinho@ufabc.edu.br 


\begin{abstract}
Renal injury caused by renal ischemia and reperfusion is capable to change heart morphology, electrophysiology, and redox unbalance. The so-called cardio-renal syndrome is an important class of dysfunction since heart and kidneys are responsible for hemodynamic stability and organ perfusion through a complex network. In the present work we investigate the Fourier-Transform Raman vibrational spectral features of cardiac hypertrophy induced by renal ischemic reperfusion. C57BL/6J mice were subjected to unilateral occlusion of the renal pedicle for 60 minutes and reperfused for 5 days, 8 days, and 15 days. It was observed that bands around 540, 1100, 1300, 1450, 1650, and 2500 $\mathrm{cm}^{-1}$ dominates the spectra. They are associated to stretching of S-S in Cysteine amino acid, stretching of $\mathrm{C}-\mathrm{C}$ in lipids, twisting of $\mathrm{CH}_{2}$ in collagen and phospholipids, bending modes of $\mathrm{CH}_{3}$ in lipids and amino acids side chains, Amide I vibration of proteins. The intensities of these vibrations are modulated during renocardiac syndrome. We find that tyrosine, tryptophan, cystine/cysteine, fibroblast growth factors, collagen III alterations from homeostasis were the metabolites associate with these changes. These findings are clinically relevant once the presented bands can be used as molecular markers of preventing cardiac diseases' development in patients with renal injury.
\end{abstract}




\section{INTRODUCTION}

The cardiorenal syndrome (CRS) is a systemic condition which heart and kidneys interact in a way that an acute or chronic injury caused in one generates a pathology in the other organ.[1] It could be classified into five main types. Types 1 and 2 correspond to the cardiorenal syndrome itself, when some injury in heart leads to a kidney injury as consequence; types 3 and 4 (also called renocardiac syndrome) an injury in kidney that leads to a heart injury; and type 5 when a systemic condition leads to a simultaneous heart and kidney injury (e.g., diabetes and sepsis).[1] All types share the inflammation as key cause.[2]

It is already known that CRS type 3 can induce cardiac injuries, being the most studied by our group, the cardiac hypertrophy. It has already been observed that the renal injury caused by renal ischemia and reperfusion (IR) is capable to change heart morphology,[3] electrophysiology,[4] and redox unbalance[5] with participation of immune system.[5]

Biophotonics techniques as Raman spectroscopy have been successfully employed to probe biological samples since they provide multiplexing quantitative and qualitative pieces of information at the molecular level even when gated by subtle changes in a sample.[6] This technique has been used in cells, tissues and biofluids studies presenting valuable findings related to pathologies.[7-9] Outstanding results concerning cardiac tissues were also reported on literature (see, e.g.,[10]). For example, Nishiki-Muranish et al.[11] analyzed Raman spectra of myocardial infarction and its repair in rats using the hypothesis that the myocardium in the course of myocardial infarction and its repair could be recognized by spontaneous Raman spectroscopy on the basis of chemical changes in myocardial tissues. Normal, necrotic, granulation, and fibrotic tissues, where discriminated with cross-validated sensitivities of 99.3, 95.3, 96.4, and 91.3\% and specificities of 99.4, 99.5, 96.5, and 98.3\% respectively. The authors shown that spontaneous Raman spectroscopy combined with partial 
least squares-discriminant analysis (PLS-DA) could be used as a novel label-free method of evaluating myocardial infarction and its repair.

Yamamoto et al.[12] identified key signatures of Raman spectra for the evaluation of myocardial viability by evaluating the infarct border zone myocardium that were excised from five patients under surgical ventricular restoration. They were able to obtain a prediction model to differentiate the infarcted myocardium from the non-infarcted myocardium by applying PLS-DA to the Raman spectra. Ohira et al.[13] tested the hypothesis whether Raman spectroscopy is applicable for evaluating myocardial ischaemia especially during early ischaemic phase. They obtained spontaneous Raman spectra of the sub-epicardial myocardium in the Langendorff-perfused rat heart upon 532-nm excitation before and during the "stopped-flow," global ischaemia. They showed that sequential measurements of the peak intensities at 750 and $1127 \mathrm{~cm}^{-1}$ enabled early detection of the myocardial ischaemia based on the mitochondrial functions.

Notwithstanding the relevance of the cited works, applications in the context of CRS are absent, to the best of our knowledge. Considering that renal ischemia and reperfusion is capable of several physiological alterations, the focus of this study was to characterize the vibrational pattern of the molecules on cardiac and renal tissues. The spectral variations induced by reperfusion were investigated and their biochemical correlations presented and discussed.

\section{MATERIALS AND METHODS}

\subsection{Animal model of renal ischemia and reperfusion (IR)}

The experiments were carried out followed the Brazilian federal law $n^{0} 11.794$, of 2008, which regulates the use of animals in scientific experimentation, according to protocol number 2728130318, approved by the Research Ethics Committee of the Universidade Federal do ABC. The 
study was carried out in compliance with the Animal Research: Reporting of In Vivo Experiments (ARRIVE) guidelines. Male C57BL/6 mice, aged between 6 and 8 weeks weighing between 20 and $25 \mathrm{~g}$, were used. All animals were housed in collective cages, containing a maximum of five animals, with a 12-hour light/dark artificial cycle, at a constant ambient temperature of $25^{\circ} \mathrm{C}$, with water and food supplement available at all times.

The protocol used was the same described on ref. [5]. The animals were submitted to anesthesia with xylazine and ketamine intraperitoneally, respectively at a dose of $10 \mathrm{mg} / \mathrm{kg}$ and 80 $\mathrm{mg} / \mathrm{kg}$ of weight diluted in $0.9 \%$ saline, single dose. The access to the renal pedicle was made through the opening of the abdominal cavity, the organs were then bounced in hydrophilic gases. After locating the left renal pedicle of the animal, all the adipose tissue from the region was removed and the left pedicle was isolated using forceps. The renal pedicle was then occluded using microvascular clips. After placing the clamp on the renal pedicle, an immediate change in kidney color could be observed due to occlusion of the local circulation, indicating the efficiency in inducing renal ischemia. The animals were clamped and kept in a thermal blanket for 60 minutes. After 60 minutes, the clamps were removed, and the viscera were replaced in the abdominal cavity with the use of flexible nails. The animals first had their peritoneum and skin sutured using a 4-0 silk thread.

After the surgical process, the animals were kept under reperfusion for 8 and 15 days. Given that, the animals were divided into three groups: SHAM (mice that had the surgical process, except the occlusion of the renal pedicle; control), 8D (mice that had the surgical procedure of occlusion of the left renal pedicle for 60 minutes and reperfusion for 8 days) and 15D (mice that had the surgical procedure of occlusion of the left renal pedicle for 60 minutes and reperfusion for 15 days). After reperfusion time, the animals were euthanized by blood extraction through the inferior vena cava. The heart and left kidneys were collected for biopsies. 


\subsection{Fourier-Transform Raman (FT-Raman) spectroscopy}

Left kidney and heart biopsies samples were submitted to vibrational spectroscopy in order to observe chemical changes in the molecular level of the heart in relation to the kidney in the days after renal ischemia induction. The FT-Raman multiraman spectrometer (Bruker Optics) operating at 1064 nm was used to spectra acquisition. Each biopsied tissue of previously thawed at room temperature was placed in an aluminum sample holder. The measurements were performed at three different points. Then, 32 cycles of scans were performed per sample using $200 \mathrm{~mW}$ of laser power. All spectra were pre-processed to become comparable for the statistical analysis. The baseline was corrected using the least-squares polynomial curve fitting method as described by Lieber and Mahadevan-Jansen in ref. [14]probabilistic quotient normalization (iqr) method was used to normalize all spectra [15] After then the classical Principal Components Analysis (PCA) and hierarchical cluster analysis (HCA) [16] were performed to extract the main spectral features discriminating experimental groups. The PCA was computed using Pareto scaling. The HCA was performed using complete clustering and euclidean distance. The correlation of each wavenumber variable with the particular scores against the covariance of that frequency variable with the same score was also plotted to find the main wavenumbers contributing to discrimination. All the spectral analysis steps were implemented in the ChemSpec vignette available in the software R.[17]

\section{RESULTS AND DISCUSSION}

Figure 1 shows the Raman spectra for heart (left) and kidney (right) tissues (groups SHAM, 8D, and 15D) in the $800-2500 \mathrm{~cm}^{-1}$ spectral window. Black lines are the average spectra while the gray vertical lines are the standard deviation of intensities. At first glance, bands around 540, 1100, 1300, 1450, 1650, and $2500 \mathrm{~cm}^{-1}$ dominates the spectra. They are associated to stretching of S-S in Cysteine amino acid, stretching of C-C in lipids, twisting of $\mathrm{CH}_{2}$ in collagen and phospholipids, bending modes of $\mathrm{CH}_{3}$ in lipids and amino acids side chains, Amide I vibration of proteins, and 
oxidation of fatty acids, respectively.[18-21] The assignment for the most relevant vibrational bands in our case is presented on Table 1.

Table 1 - Assignment of vibrational modes, based on refs [18-21].

\begin{tabular}{|c|c|}
\hline Wavenumber $\left.\mathbf{c m}^{-\mathbf{1}}\right)$ & Assignment \\
\hline 520 & S-S disulfide stretching in cystine or cysteine amino acids \\
\hline 625 & C-C twisting mode of phenylalanine \\
\hline 770 & Ring breathing tryptophan \\
\hline 1430 & Deoxyribose in B or Z DNA conformation \\
\hline 1450 & Bending modes of methyl groups (collagen) \\
\hline 1605 & Cytosine (NH $\left.\mathrm{N}_{2}\right)$ \\
\hline 1615 & C=C (Tyrosine or tryptophan) \\
\hline 1655 & Amide I ( $\alpha$-helix of collagen) \\
\hline 2500 & Oxidation of fatty acids \\
\hline
\end{tabular}

After computing PCA one conclude that 1st and 3rd components present the largest set of spectral features enabling discriminate SHAM, 8D, and 15D groups for heart. The dispersion plot of PC3 against PC1 (top of Fig. 2) represents this separation. An almost complete separation between SHAM and 15D groups is observable. The dendrogram using PC1 and PC3 scores for heart samples is shown Fig. 3 (top). The data clustered on two great groups. Cluster 1 is composed by almost all 15D samples. Some mixing with 2 8D samples was also observed. Once excluding outliers (cluster *), all SHAM samples are present on cluster 2 jointly with almost all 8D samples. Thus cluster 2 represents SHAM and 8D classes. It is possible state that a complete discrimination was observed only for $15 \mathrm{D}$ class (cluster 1 ) for heart tissues. For kidney tissue the 1st and 2nd components are responsible for the largest set of spectral characteristics discriminating experimental groups (Fig. 2 bottom). In this case the greatest discrimination was observed for SHAM class of samples. The 
dendrogram for kidney (bottom of Fig. 3) indicates that 8D and 15D samples clustered on sample group (cluster 1) while SHAM is $100 \%$ present on cluster 2.

For kidney tissues the 1st and 2nd components were responsible for the largest set of spectral characteristics discriminating experimental groups (bottom of Fig. 3). In this case the greatest discrimination was observed for SHAM class of samples. The dendrogram for kidney (bottom of Fig. 3) indicates that $8 \mathrm{D}$ and $15 \mathrm{D}$ samples clustered on sample group (cluster 1) while SHAM is $100 \%$ present on cluster 2 .

By inspection of plot of correlation of each wavenumber variable with the particular score against the covariance of that wavenumber variable with the same score (s-plot) one is able to find those bands influences that score the most. The s-plot has the most influential variables in the upper right hand and lower left quadrants. Figure 4 presents the s-plot for heart (PC1 and PC3, top) and kidney (PC1 and PC2, bottom). Bands at 625, 1615, and $1655 \mathrm{~cm}^{-1}$ presented the greatest contributions to 1st and 3rd scores for heart. In kidney case, bands at 520, 770, 1430, 1450, and 1605 $\mathrm{cm}^{-1}$ influenced 1st and 2nd scores the most. The loading plots for heart (Fig. 5, top) and kidney (Fig. 5, bottom) confirmed the findings observed on s-plot.

Figures 6 shows the heatmaps of most influential bands for heart. The $8 \mathrm{D}$ class presented a significant enhancement in the $625 \mathrm{~cm}^{-1}$ band intensity compared to the others. This band corresponds to $\mathrm{C}-\mathrm{C}$ twisting mode of phenylalanine amino acid. The $1615 \mathrm{~cm}^{-1}$ band is assigned to $\mathrm{C}=\mathrm{C}$ stretching of tyrosine or tryptophan and also displayed significant intensity increasing for 8D samples. Interestingly, for the $1655 \mathrm{~cm}^{-1}$ band it was observed that 8D and some SHAM samples clustered together in the lowest intensity cluster in bottom. This band is associate to Amide I vibration. This wavenumber is related to $\alpha$-helix secondary structure of collagen.

The heatmaps for kidney tissues are presented on Fig. 7. The $520 \mathrm{~cm}^{-1}$ band associated to S-S disulfide stretching in cysteine and cistine amino acids presented distinctive decreasing intensity on 
$15 \mathrm{D}$ group. SHAM and 8D classes merged on same cluster. For $770 \mathrm{~cm}^{-1}$ band (ring breathing of tryptophan) it was observed that SHAM group has the lowest intensity while 8D and 15D clustered together on same group on dendrogram. Similar behavior was observed for $1430 \mathrm{~cm}^{-1}$ band which is assigned as deoxyribose (marker for $\mathrm{B}$ or $\mathrm{Z}$ conformation). A remarkable decreasing in the $1450 \mathrm{~cm}^{-1}$ band intensity for 8D group (bending modes of methyl groups (collagen) contrasted to SHAM one as also observed. The 15D class shown an intermediate intensity level. This band is related to methyl vibrations in collagen and this result indicates decreasing methylation on collagen protein for 8D. Also, remarkable was the observed decreasing in the $1605 \mathrm{~cm}^{-1}$ band $\left(\mathrm{NH}_{2}\right.$ bending in Cytosine) for 15D compared to SHAM group.

We were able to observe that amino acid metabolism was the most perturbed by metabolic pathways in this model of renal ischemia and reperfusion. The discovery of increased tyrosine/ tryptophane after 8 days of reperfusion (8D) seems to have important physiological consequences. These amino acids have already been studied in chronic and acute kidney injuries. A tyrosine blood drop has been earlier observed during chronic kidney disease and was explained as an impaired synthesis of tyrosine from phenylalanine, by the kidney.[22] Besides, the main role of the kidney is blood filtration, in order to eliminate waste products resulting from the metabolism (e.g. urea, creatinine, uric acid, indoxyl sulfate). Kidney failure can be characterized by an accumulation of toxic compounds in the body, some of these metabolites have a well-documented toxicity and are referred to as uremic toxins (UT). Our results showed that it is possible to identify molecular markers such tyrosin and tryptophane which are the main raw materials of the protein-bounded UTs. The progression of acute to chronic kidney injury is proportional to the quantity of UTs through circulation.[23]

Fibrosis is another relevant physiological point raised on the present study. After 15 days (15D) we have observed an increasing of the band associated to Amide I vibration in heart tissue, 
related to $\alpha$-helix secondary structure of collagen. Our previous results indicated the lack of fibrosis in the heart tissue.[3] Physiologically, the non-deposition of collagen does not mean that this gene is not been regulated as well as the increase in some fibroblast growth factors (FGF). Some of these FGFs are responsible not only for the heart injury but also for the kidney disease progression. One example is FGF23 which is responsible for increase in phosphate levels, aggravating the acute renal injury.[24] FGF23 itself causes hypertrophy left ventricular.[25] Thus we argue that the observed increase in collagen content in cardiac tissue is related to hypertrophic factors activated by collagen but without its deposition.

In kidney tissue, after 15 days (15D) we find a decreasing in the band associated to collagen III (cysteine and cistine amino acids bands) in the tissue. Renal IR main consequence is renal fibrosis. [26]ype III collagen is secreted by fibroblasts and other mesenchymal cell types, thus making it a major player in various inflammation-associated pathologies as, e.g., kidney fibrosis.[27] Its serum concentration usually increases in the fibrosis. Thus, collagen III content in the tissue is expected to decrease.

The inflammation in the left kidney is already target of our studies.[5] The inflammation in this model of IR is possible initiated by the oxidative stress and enlarged by the NO release, already observed after 8 days of reperfusion comparing data of protein oxidation and superoxide dismutase levels.[28] Oxidative stress is the balance between production of free radicals and reduced antioxidant defenses. It is often increased by inflammation and mitochondrial dysfunction.[29] The oxidative stress works like a gear, activating the transforming growth factor $\beta 1$ (TGF- $\beta 1$ )[29] that are able to induce fibrosis in the kidney in part by activating NADPH oxidases.[30] A better understanding of the signaling pathways by which oxidative stress induces renal fibrosis may lead to the development of novel therapeutic strategies. 
Trentin-Sonoda et al.[5] pointed an atrophy of the left kidney after IR, based on kidney morphometric data and magnetic resonance imaging. Kidney weight starts to decrease around the 8th day of reperfusion and persists until 15th day and remains smaller even after 8 weeks of reperfusion (data not published). However, the opposite happens to the inflammatory factors: it normalizes after 8 days of IR, as well as the kidney function. However, it probably means that kidney function can be normalized once there is a contra-lateral kidney working normally (right kidney) but the injured kidney (left kidney submitted to occlusion) may have a scar due to the fibrosis process induced by the ischemic injury. In a chronic kidney insufficiency or aging, for example, it is possible to observe that normal kidney may be overloaded and unable to maintain control of hemodynamic balance, leading to other cardiac changes. In this sense, the collagen levels in the kidney combined to a S-S disulfide stretching in cysteine and cistine amino acids can mean an important marker of kidney injury, and a predictor of cardiovascular changes observed in CRS 3.

\section{CONCLUSIONS}

Our results shown that intensities of vibrations associated to stretching of S-S in Cysteine amino acid, stretching of C-C in lipids, twisting of $\mathrm{CH}_{2}$ in collagen and phospholipids, bending modes of $\mathrm{CH}_{3}$ in lipids and amino acids side chains, Amide I vibration of proteins, are modulated during CRS type 3 induced in vivo by renal ischemia and reperfusion. Tyrosine, tryptophan, cystine/cysteine, fibroblast growth factors, and collagen III alterations from homeostasis were the metabolites changes associate with these vibrational changes. These findings are clinically relevant once this bands can be used as molecular markers related to cardiac diseases development in patients with renal injury. Finally, the molecular signatures found as markers of uremic compounds can represent a major clinical finding for acute or chronic kidney diseases and consequently predict future heart diseases. 


\section{ACKNOWLEDGMENTS}

The authors would like to thank the Brazilian agencies Conselho Nacional de Desenvolvimento Científico e Tecnológico (CNPq - 311146/2015-5 and 307718/2019-0) and Fundação de Amparo á Pesquisa do Estado de São Paulo (FAPESP - 2011/19924-2) for the financial support. The authors would also thank the experimental resources provided by Multiuser Central Facilities at UFABC (CEM/UFABC).

\section{REFERENCES}

[1] Lullo, L. D. et al. Pathophysiology of the cardio-renal syndromes types 1-5, An uptodate. Indian Heart J. 69, 255-265 (2017).

[2] Colombo, P. C. et al. Inflammatory activation, cardiac, renal, and cardio-renal interactions in patients with the cardiorenal syndrome. Heart Failure Rev. 17, 177-190 (2011).

[3] Cirino-Silva, R. et al. Renal ischemia/reperfusion-induced cardiac hypertrophy in mice. Cardiac morphological and morphometric characterization. JRSM Cardiovasc. Dis. 6, 204800401668944 (2017).

[4] Alarcon, M. M. L. et al. Cardiac arrhythmias after renal I/R depend on IL-1upbeta. J. Mol. Cell. Cardiol. 131, 101-111 (2019).

[5] Trentin-Sonoda, M. et al. Knockout of Toll-Like Receptors 2 and 4 Prevents Renal IschemiaReperfusion-Induced Cardiac Hypertrophy in Mice. PLOS One 10, e0139350 (2015).

[6] Baker, M. J. et al. Clinical applications of infrared and Raman spectroscopy, state of play and future challenges. Analyst 143 , 1735-1757 (2018).

[7] Jermyn, M. et al. Intraoperative brain cancer detection with Raman spectroscopy in humans. Sci. Transl. Med. 7, 274ra19.(2015).

[8] Lin, D. et al. Autofluorescence and white light imaging-guided endoscopic Raman and diffuse reflectance spectroscopy for in vivo nasopharyngeal cancer detection. J. Biophotonics 11 , e201700251.(2018)

[9] Mitropoulos, K., Katsila, T., Patrinos, G. P. \& Pampalakis, G. Multi-Omics for Biomarker Discovery and Target Validation in Biofluids for Amyotrophic Lateral Sclerosis Diagnosis. OMICS 22 , 52-64.(2018). 
[10] Chaichi, A., Prasad, A. and Gartia, M. Raman Spectroscopy and Microscopy Applications in Cardiovascular Diseases, From Molecules to Organs. Biosensors 8 , 107.(2018).

[11] Nishiki-Muranishi, N. et al. Label-Free Evaluation of Myocardial Infarction and Its Repair by Spontaneous Raman Spectroscopy. Anal. Chem. 86 , 6903-6910.(2014).

[12] Yamamoto, T. et al. Label-free Evaluation of Myocardial Infarct in Surgically Excised Ventricular Myocardium by Raman Spectroscopy. Sci. Rep. 8.(2018).

[13] Ohira, S. et al. Label-free detection of myocardial ischaemia in the perfused rat heart by spontaneous Raman spectroscopy. Sci. Rep. 7.(2017).

[14] Lieber, C. A. and Mahadevan-Jansen, A. Automated Method for Subtraction of Fluorescence from Biological Raman Spectra. Appl. Spectrosc. 57 , 1363-1367.(2003).

[15] Dieterle, F., Ross, A., Schlotterbeck, G. \& Senn, H. Probabilistic Quotient Normalization as Robust Method to Account for Dilution of Complex Biological Mixtures. Application in1H NMR Metabonomics. Anal. Chem. 78, 4281-4290.(2006).

[16] Varmuza, K. \& Filzmoser, P., Introduction to multivariate statistical analysis in chemometrics. CRC press, .2016.

[17] Team, R. C. et al. R, A language and environment for statistical computing, . (2013).

[18] Bitar, R. A., da Silva Martinho, H., Tierra-Criollo, C. J., Ramalho, L. N. Z., Netto, M. M. \& Martin, A. A. Biochemical analysis of human breast tissues using Fourier-transform Raman spectroscopy. J. Biomed. Opt. 11 , 054001.(2006).

[19] Movasaghi, Z., Rehman, S.\& Rehman, I. U. Raman Spectroscopy of Biological Tissues. Appl. Spec. Rev. 42 , 493-541.(2007).

[20] Owens, G. L., Gajjar, K., Trevisan, J., Fogarty, S. W., Taylor, S. E., Gama-Rose, B. D., MartinHirsch, P. L. \& Martin, F. L. Vibrational biospectroscopy coupled with multivariate analysis extracts potentially diagnostic features in blood plasma/serum of ovarian cancer patients. J. Biophotonics 7 , 200-209.(2013).

[21] Rodrigues, L. M., Alva, T. D. M., da Silva Martinho, H. \& Almeida, J. D. Analysis of saliva composition in patients with burning mouth syndrome (BMS) by FTIR spectroscopy. Vib. Spectrosc. 100, 195-201.(2019).

[22] Kopple, J. D. Phenylalanine \& Tyrosine Metabolism in Chronic Kidney Failure. J. Nutr. 137 , 1586S-1590S.(2007). 
[23] Fabresse, N., Uteem, I., Lamy, E., Massy, Z., Larabi, I. A. \& Alvarez, J.-C. Quantification of free and protein bound uremic toxins in human serum by LC-MS/MS, Comparison of rapid equilibrium dialysis and ultrafiltration. Clin. Chim. Acta 507 , 228 - 235.(2020).

[24] Edmonston, D. \& Wolf, M. FGF23 at the crossroads of phosphate, iron economy and erythropoiesis. Nat. Rev. Nephrol. 16 , 7-19.(2019).

[25] Faul, C. et al. FGF23 induces left ventricular hypertrophy. J. Clin. Investig. 121 , 4393-4408. (2011).

[26] Feitoza, C. Q. et al. Inhibition of COX 1 and 2 prior to Renal Ischemia/Reperfusion Injury Decreases the Development of Fibrosis. Mol. Med. 14, 724-730.(2008).

[27] Karsdal, M., Biochemistry of collagens , structure, function and biomarkers. Academic Press, London, United Kingdom.2016.

[28] Caio-Silva, W. et al. Characterization of the Oxidative Stress in Renal Ischemia/ReperfusionInduced Cardiorenal Syndrome Type 3. BioMed Res. Int. 2020 , 1-11.(2020).

[29] Lv, W., Booz, G. W., Fan, F., Wang, Y. \& Roman, R. J. Oxidative Stress and Renal Fibrosis, Recent Insights for the Development of Novel Therapeutic Strategies. Front. Physiol. 9.(2018).

[30] Baltanás, A. et al. A Synthetic Peptide from Transforming Growth Factor-upbeta1 Type III Receptor Inhibits NADPH Oxidase and Prevents Oxidative Stress in the Kidney of Spontaneously Hypertensive Rats. Antioxid. Redox Signal. 19 , 1607-1618.(2013). 


\section{Figures}

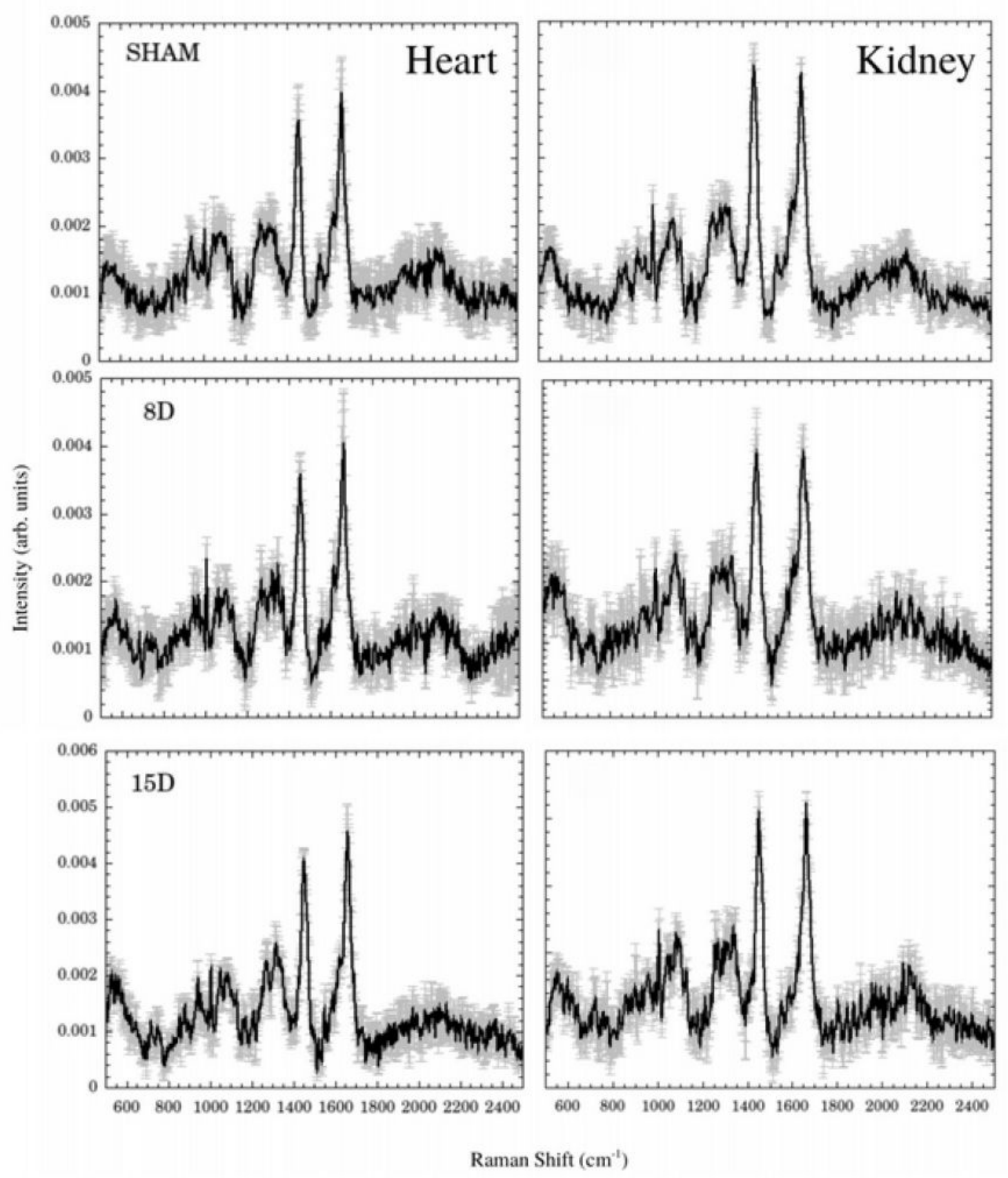

Figure 1

Average Raman spectra (black line) for heart (left) and kidney (right) tissues for SHAM, 8D, and 15D groups. The vertical gray lines are the standard deviation. 

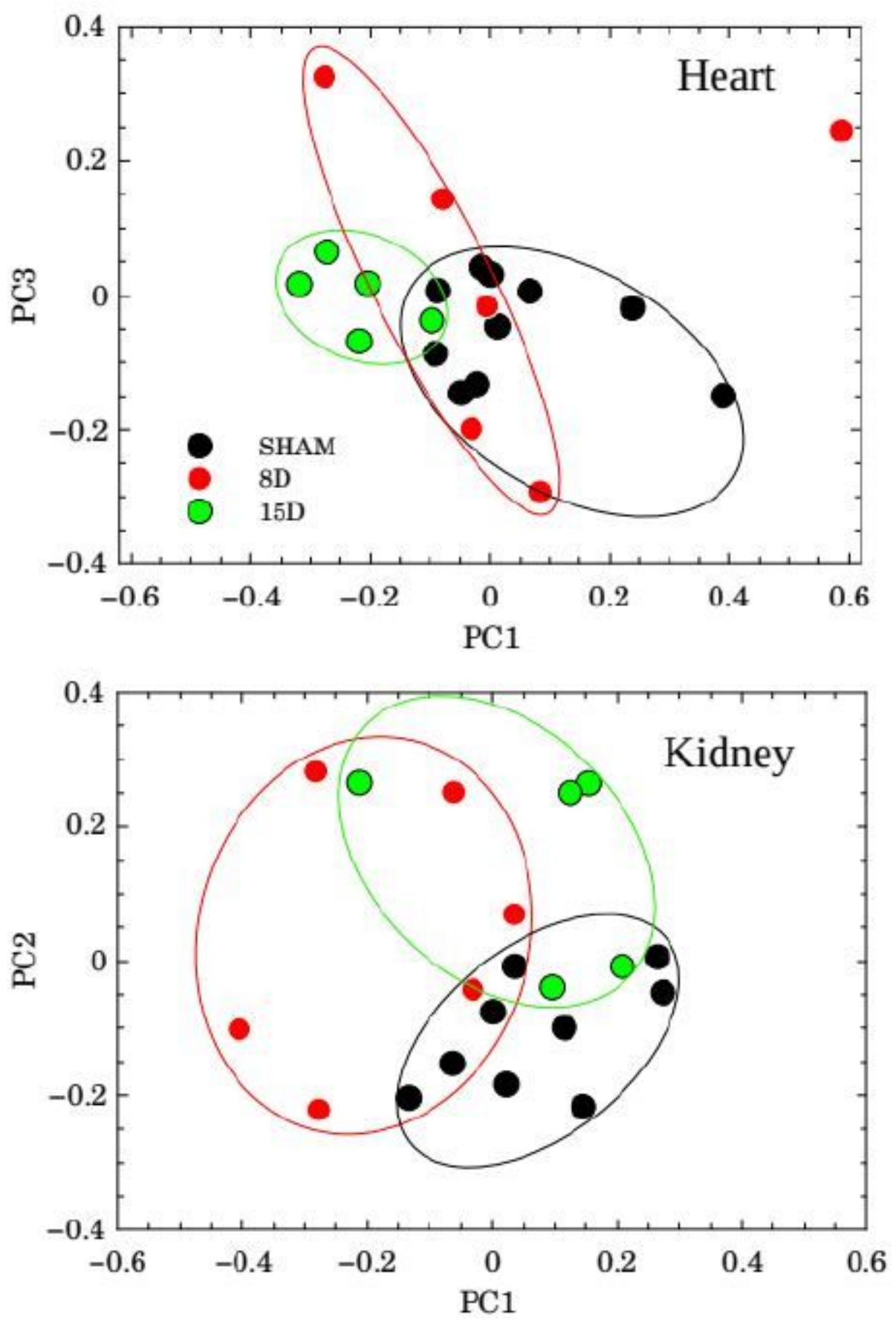

Figure 2

Dispersion plot of PCs for heart (top) and kidney (bottom) tissues. Black, red and green symbols indicates the SHAM, 8D, and 15D groups, respectively. Classical confidence ellipses are also included for each group. 

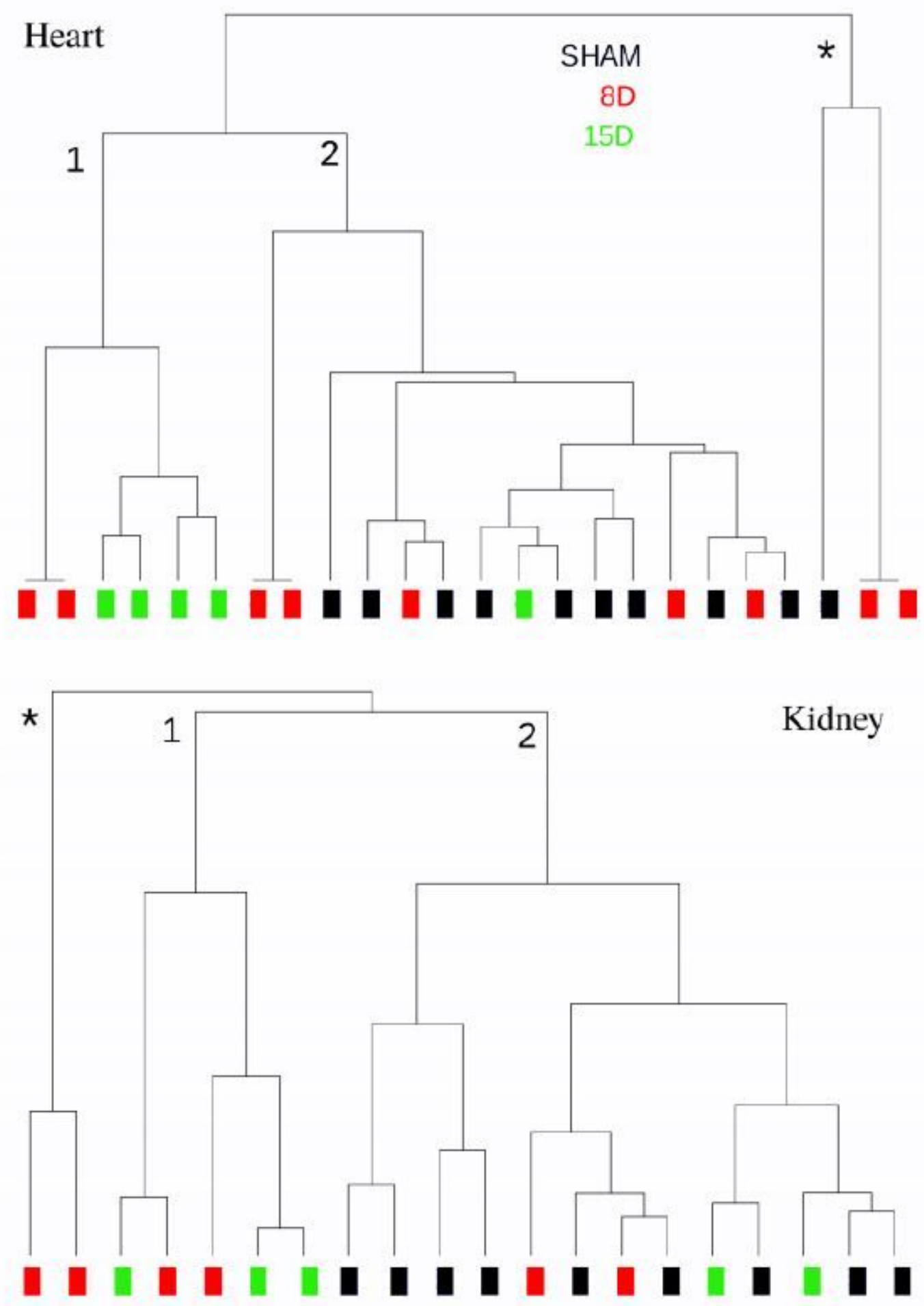

\section{Figure 3}

Dendrogram from hierarchical clustering (complete clustering, euclidean distance) for heart (top) and kidney (bottom) tissues. First and 3rd PC scores were used for heart tissue while for kidney the 1st and 2nd PC scores were employed. Blue, red and green symbols indicate the SHAM, 8D, and 15D groups, respectively. 


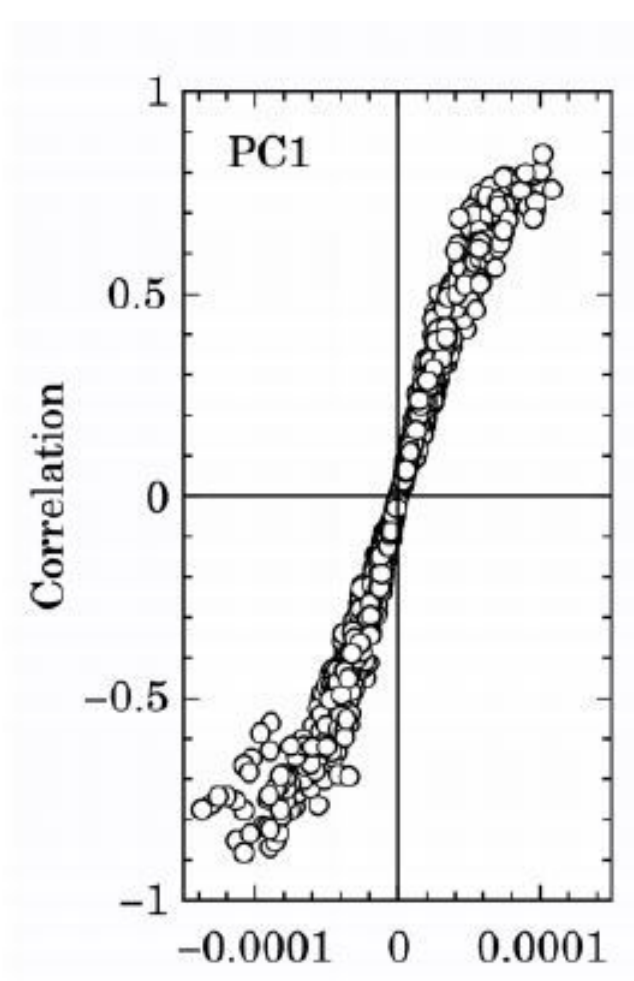

Heart

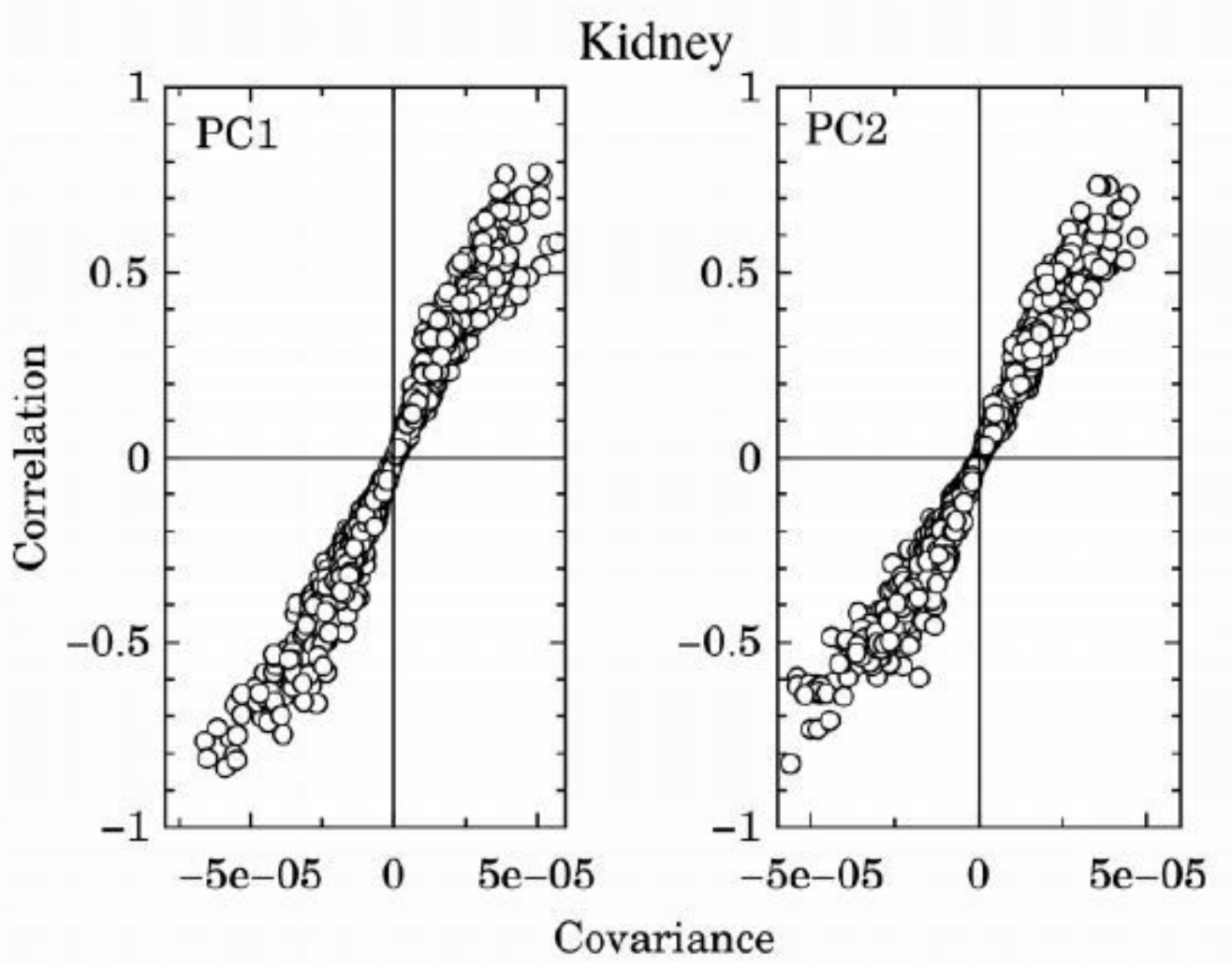

Figure 4

Correlation vs Covariance plot for heart (top) and kidney (bottom) tissues. 


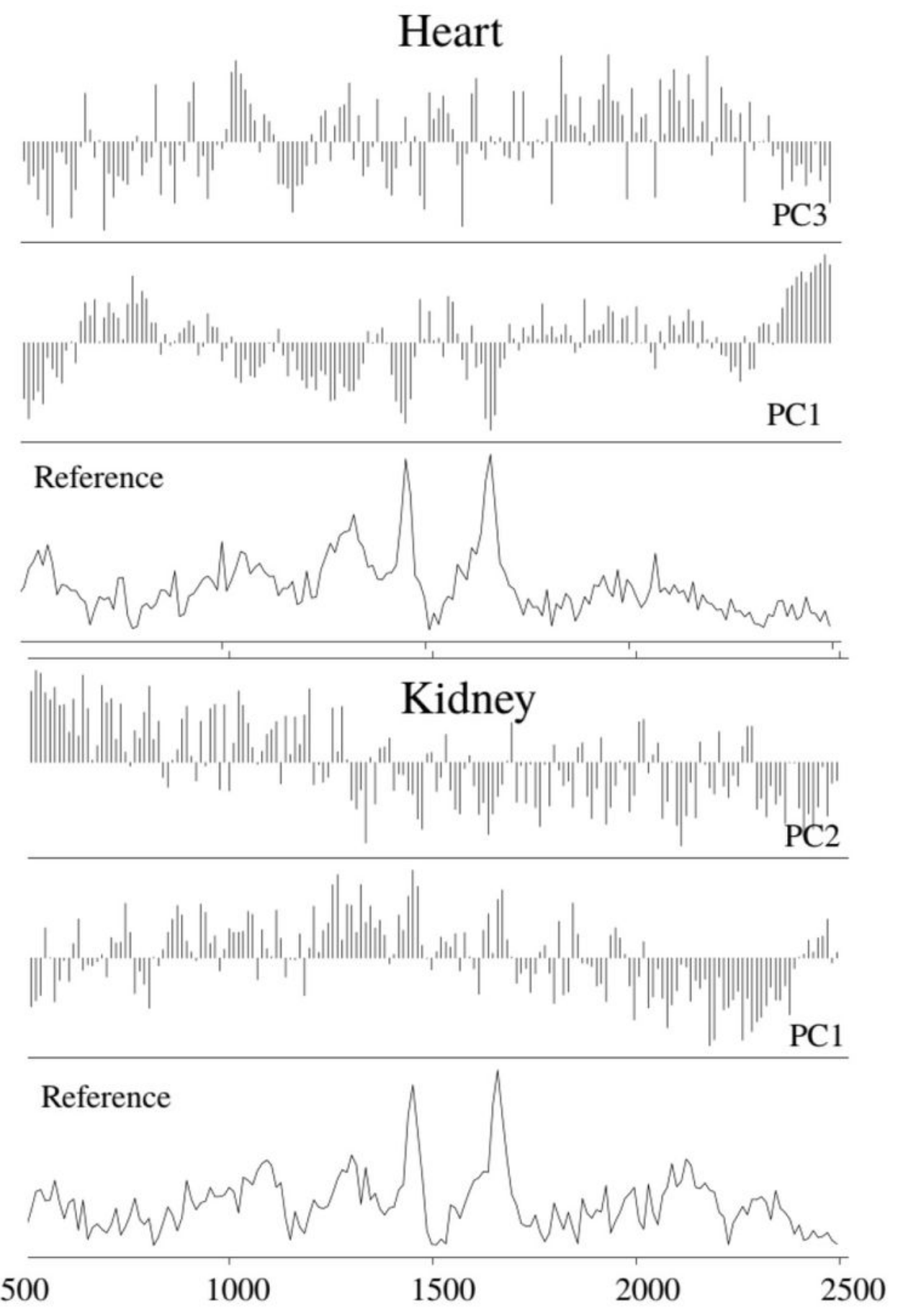

Figure 5

Loading plots for heart (PC1,PC3, on top) and kidney (PC1, PC2, on bottom) tissues. The average spectra each kind of tissue is shown for reference. 


\section{HEART}
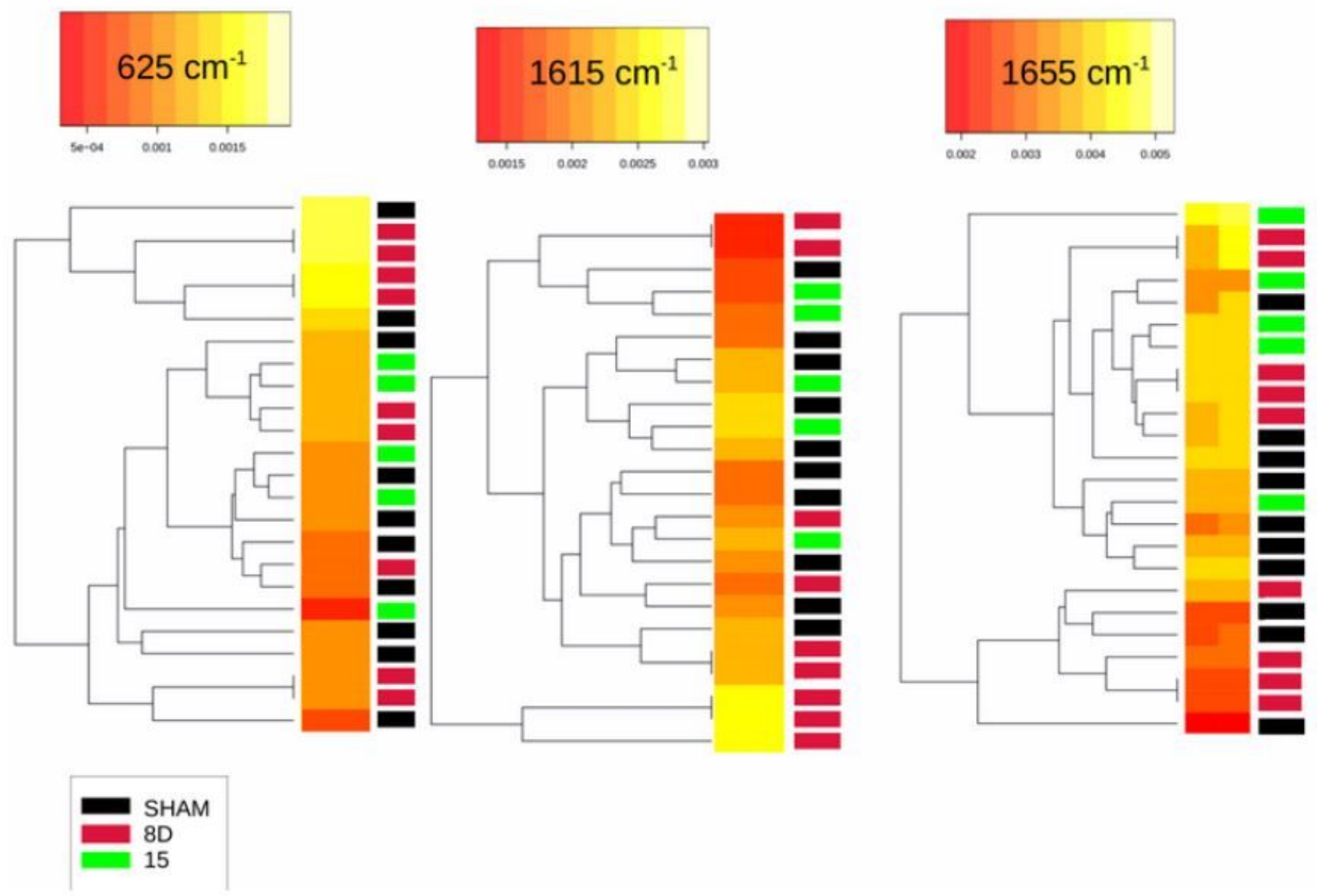

Figure 6

Heatmaps for $625,1615,1655$, bands in heart samples. 


\section{KIDNEY}
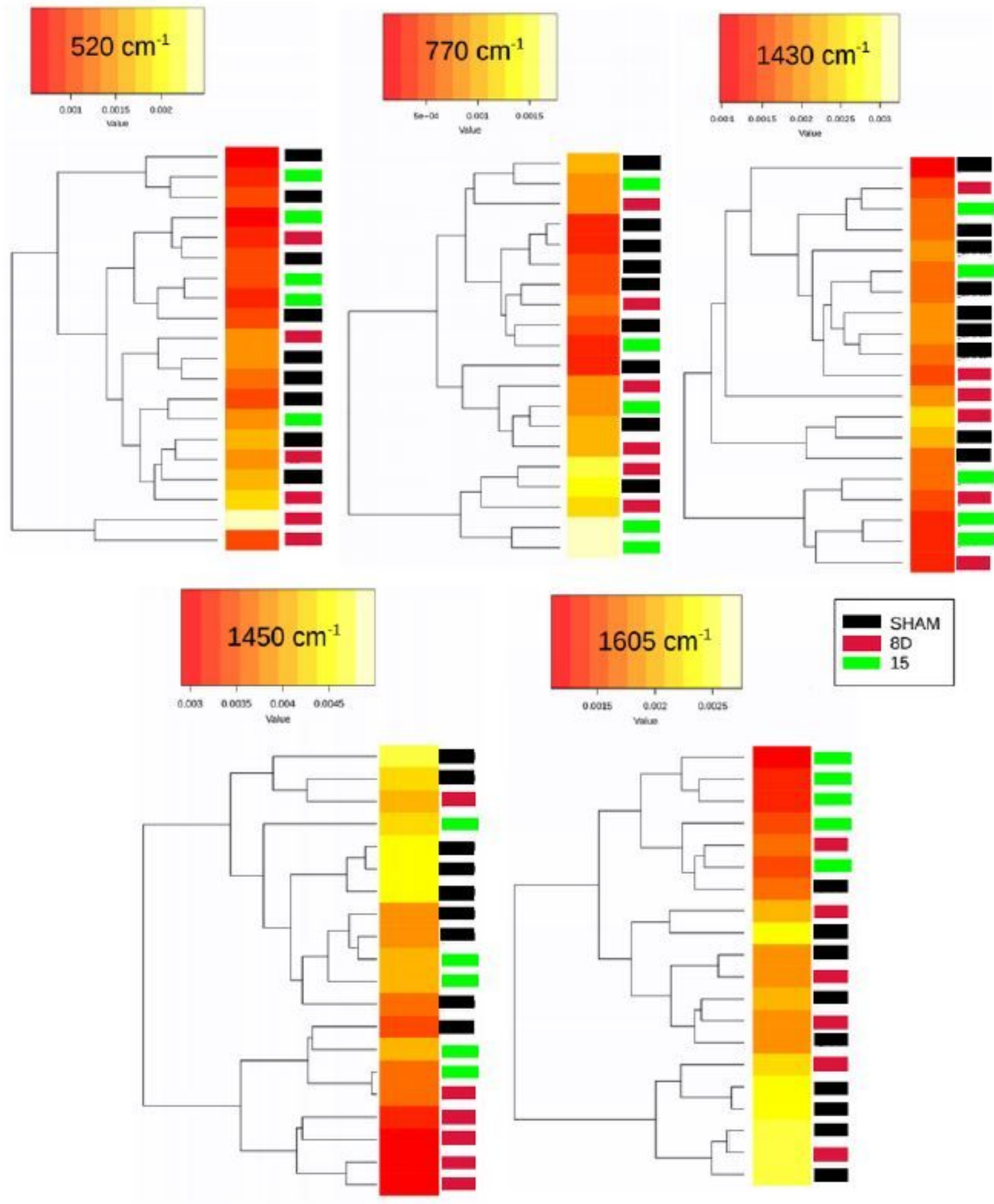

Figure 7

Heat-maps for $520,770,1430,1450$, and $1605 \mathrm{~cm}-1$ bands in kidney samples. 